\title{
Modelling and Simulation of Laser Welded Joints of Reinforced PP and PC Plastics
}

\author{
Vlastimil Votrubec ${ }^{1}$ \\ ${ }^{1}$ VÚTS, a.s., Liberec, Czech Republic \\ Correspondence: Vlastimil Votrubec, VÚTS, a.s., Svárovská 619, 460 01, Liberec, Czech Republic. Tel: \\ 420-485-302-387. E-mail: vlastimil.votrubec@vuts.cz
}

Received: August 17, 2018

Accepted: October 12, $2018 \quad$ Online Published: January 10, 2019

doi:10.5539/mer.v9n1p1

URL: https://doi.org/10.5539/mer.v9n1p1

\begin{abstract}
Welding of composites decreases their material strength. Proper evaluation of FEM simulations is necessary for correct design and safety factor determination. Neat and reinforced PP and PC specimens were measured on a testing machine to obtain their material properties. Experimental tensile curves were used as inputs in FEM simulations. 15 combinations of specimen were welded on laser work machine and afterwards the welded joint strength was measured on the testing machine. The results of shear tests and FEM simulations are compared in this paper. Also the influence of fiber presence in composites on welded joint strength is discussed. At the end suggestions for safe machinery design of laser welded reinforced composites are given.
\end{abstract}

Keywords: laser welding, reinforced plastics, FEM simulations, welded joints

\section{Introduction}

Welding of materials has a big influence on its structure. During the laser welding process the jointed materials at contacting surfaces are heated while both materials are melted and through intermolecular diffusion united. The indivisible joint is created after cooling. As a result, the residual thermal stress is present and the material structure can be changed (Papazoglou et al., 1982; Colegrove et al., 2009). The structure modification is distinct especially if the origin material has complicated structure, e.g. reinforced plastics. Also, there is often a notch in the joint which causes additional concentration of stress. Therefore, the strength of welded joint is supposed to be lower compared to the material strength itself.

Various welding technologies for thermoplastic composites were developed in the last 20 years (da Costa et al., 2012; Grewell \& Benatar, 2007), e.g. ultrasonic welding (Kumar et al., 2016), resistance welding (Stavrov \& Bersee, 2005) or laser welding (Al-Wohoush \& Kamal, 2012). Research in this area is usually focused on welded process itself: heat transfer during the welding, phase transition, changes of structure, analysis of residual stress, welding conditions necessary for quality welds, welding of different materials etc. The process of welding can be also described by numerical simulations using the finite elements method (Yaghi \& Becker, 2004; He, 2012; Mackerle, 2001). The outputs of analysis should be used for correct design of weld joints.

This paper deals with welded joint strength of reinforced and neat polypropylene (PP) and polycarbonate (PC) polymers and possibilities of weld joint modelling, simulation and design. The finite elements simulations are adjusted to shear tests. Results of measurement and simulations are compared and some advices for safe design are given. The influence of fibers volume ratio on the weld joint strength is also discussed.

\section{Materials Used for Experiments}

Knowledge of material properties is necessary for every finite element simulation. Plastic materials are not linear so the whole tensile curve of each used material was measured on the testing machine by standard tensile test. Experimental data were processed statistically and adjusted to the proper form for sw Siemens NX. Materials used for testing welded joints by shear tests on a testing machine and computations by finite element method are in the table 1 . There are also values of their tensile strength and Poisson ratio.

Laser welding requires one material transparent so the light beam gets through to the non-transparent material working as an absorber of the light therefore on of the materials, in this paper marked by letter B (black), has to be darkened by absorber additives, usually by carbon black. These additives cause partially change of material 
properties (see the table 1). The second material, transparent, is marked by letter $\mathrm{N}$ (natural). The number following letter $\mathrm{N}$ or $\mathrm{B}$ means the amount of glass fibers in the polymer (in percentage).

Table 1. Material used for testing

\begin{tabular}{lcc|ccc}
\hline Material & $\begin{array}{c}\text { Tensile strength } \\
{[\mathrm{MPa}]}\end{array}$ & Poisson ratio & Material & $\begin{array}{c}\text { Tensile strength } \\
{[\mathrm{MPa}]}\end{array}$ & Poisson ratio \\
\hline PP N0 & 33 & 0.45 & PP B0 & 36 & 0.46 \\
PP N20 & 66 & 0.46 & PP B20 & 63 & 0.51 \\
PP N40 & 66 & 0.46 & PP B40 & 73 & 0.46 \\
PC N0 & 60 & 0.40 & PC B0 & 64 & 0.40 \\
PC N15 & 81 & 0.39 & PC B15 & 83 & 0.40 \\
PC N30 & 85 & 0.34 & PC B30 & 94 & 0.36 \\
\hline
\end{tabular}

Note. Mechanical properties (tensile strength and Poisson ratio) of polypropylene (PP) and polycarbonate (PC) composites used for testing and simulating welded joints.

\section{Modelling of Shear Test}

The simulation model was created to correspond with shear tests performed on the testing machine. The simulation sw NX Siemens was used. The finite element model consisted of two bars of the same size $20 \times 130 \times 2 \mathrm{~mm}$ and the lap length was $20 \mathrm{~mm}$ (Fig. 1). Each bar was split into several parts so the mesh could be of different sizes and the boundary conditions and the load could be applied. The volume of weld joint and close surroundings were meshed finely to express the stress changes well. The weld joint was across the entire width of the bar and it was $1 \mathrm{~mm}$ wide.

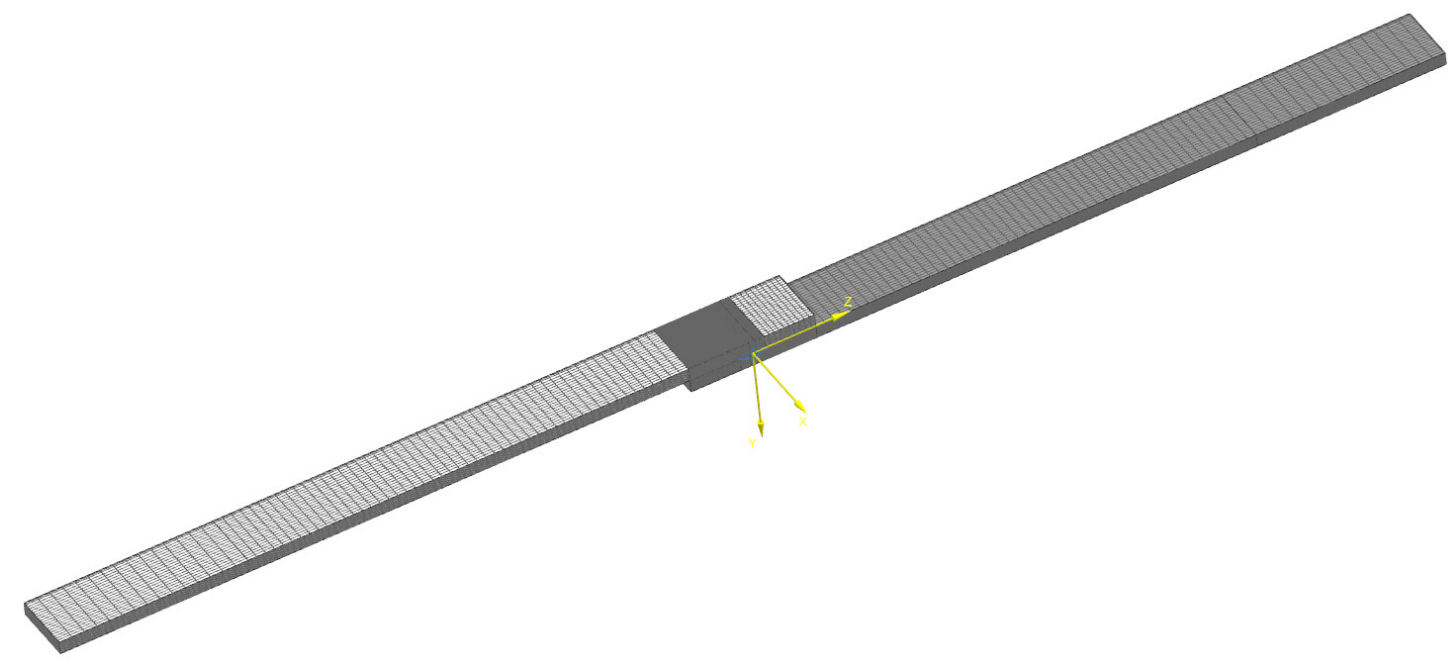

Figure 1. Finite element model of welded bars in sw NX Siemens.

According to the experiments on the testing machine the fixed constraint was applied at the end of the right bar, the load in the longitudinal direction (z-axis) was applied at the end of the left bar. The value of loaded force was derived from the maximal value obtained from the shear tests. Real welded area varied from the model and it was different for every combination so the force value had to be re-calculated according to weld area in the model. The task is symmetric so it was enough to simulate only one half of the model. The mesh consisted of quadratic hexa elements. The weld joint itself was realized by mesh mating of contacting surfaces of weld joint.

The created mesh remained the same for all combinations of welded materials (table 2). The simulation models differed in used materials and value of exerted force. The result of the simulations was a stress distribution in weld joints and surroundings. The outputs of the simulations were compared with measurements and were a subject of interpretation and discussion. 
Table 2. Combinations of welded materials for shear tests and simulations

\begin{tabular}{lcc}
\hline Material & Material & Material \\
\hline PP N0-B0 & PP N20-B0 & PP N40-B0 \\
PP N0-B20 & PP N20-B20 & PP N40-B20 \\
PP N0-B40 & PP N20-B40 & PP N40-B40 \\
PC N0-B0 & PC N15-B0 & \\
PC N0-B15 & PC N15-B15 & \\
PC N0-B30 & PC N15-B30 & \\
\hline
\end{tabular}

\section{Experiments on testing machine}

The laser working station LM05/05P.W produced continuous weld joint $1 \mathrm{~mm}$ wide across the jointed bars. Welding of PC composites was quite demanding so three combinations of materials couldn't be welded. Also the variance of measured data is larger compared to PP composites. Welding conditions, data processing, evaluation and important outputs of these tests were described earlier (Votrubec, 2017). Therefore only the results are shown in the table 3.

Table 3. Statistically processed measured data of shear tests - mean value and $95 \%$ confidence interval of maximal force, welded area and calculated maximal stress

\begin{tabular}{llll}
\hline & Maximal force $[\mathrm{N}]$ & Welded area $\left[\mathrm{mm}^{2}\right]$ & Maximal stress [MPa] \\
\hline PP N0 - B0 & $246 \pm 16$ & $14.6 \pm 1.5$ & $16.9 \pm 1.1$ \\
PP N0 - B20 & $254 \pm 11$ & $14.5 \pm 1$ & $17.6 \pm 1.2$ \\
PP N0 - B40 & $290 \pm 8$ & $19.8 \pm 0.8$ & $14.7 \pm 0.5$ \\
PP N20 - B0 & $319 \pm 22$ & $18.6 \pm 1.4$ & $17.2 \pm 1$ \\
PP N20 - B20 & $314 \pm 39$ & $17.9 \pm 1.6$ & $17.6 \pm 1.4$ \\
PP N20 - B40 & $504 \pm 56$ & $24.6 \pm 1.4$ & $20.4 \pm 1.9$ \\
PP N40 - B0 & $277 \pm 50$ & $18.6 \pm 2.6$ & $14.9 \pm 1.5$ \\
PP N40 - B20 & $476 \pm 57$ & $24.2 \pm 2.5$ & $19.6 \pm 0.7$ \\
PP N40 - B40 & $702 \pm 28$ & $36 \pm 2$ & $19.5 \pm 1.2$ \\
PC N0 - B0 & $380 \pm 80$ & $12.9 \pm 1$ & $29.9 \pm 7.5$ \\
PC N0 - B15 & $358 \pm 37$ & $15.8 \pm 1.7$ & $23.5 \pm 4.7$ \\
PC N0 - B30 & $398 \pm 28$ & $11.9 \pm 0.5$ & $33.6 \pm 2.3$ \\
PC N15 - B0 & $758 \pm 39$ & $26.2 \pm 5.8$ & $30.4 \pm 4.8$ \\
PC N15 - B15 & $685 \pm 48$ & $21.3 \pm 1.7$ & $31.1 \pm 1.9$ \\
PC N15 - B30 & $477 \pm 77$ & $18.6 \pm 0.7$ & $25.7 \pm 4.2$ \\
\hline
\end{tabular}

First numbers of maximal force, welded area and computed maximal stress denote mean values and second numbers denote $95 \%$ confidence interval.

The maximal stress in the table 3 is computed as a ratio of measured maximal force and welded area and this value means the strength of welded joint. The strength is relatively similar for all combinations of welded materials (PP and PC separately) and much lower than the material strength mentioned in the table 1 . The main conclusion of these tests can be that the weld joint strength doesn't depend on volume ratio of fibers and is approximately half to the strength of neat material.

\section{Simulation Results}

The value of loaded force corresponds to the force which caused a damage of the weld joint and thus separation of the bars. The task was to find dependence or causal link between the measured stress and results of the simulations. The distribution of stress is the same for all combinations, only the values of stress and local peaks stress varied. The overall view on von Mises stress distribution on the welded bars is in the figure 2.

Detailed evaluation of simulation results is done only for sole combination PP N0-B0. From the rest of the simulations only relevant values were chosen for comparison. The difference between stress values of "natural" and "black" bar is minimal. The most interesting part of the model is the connection area inside the bars so only the detail of this area is displayed in the following figures. 


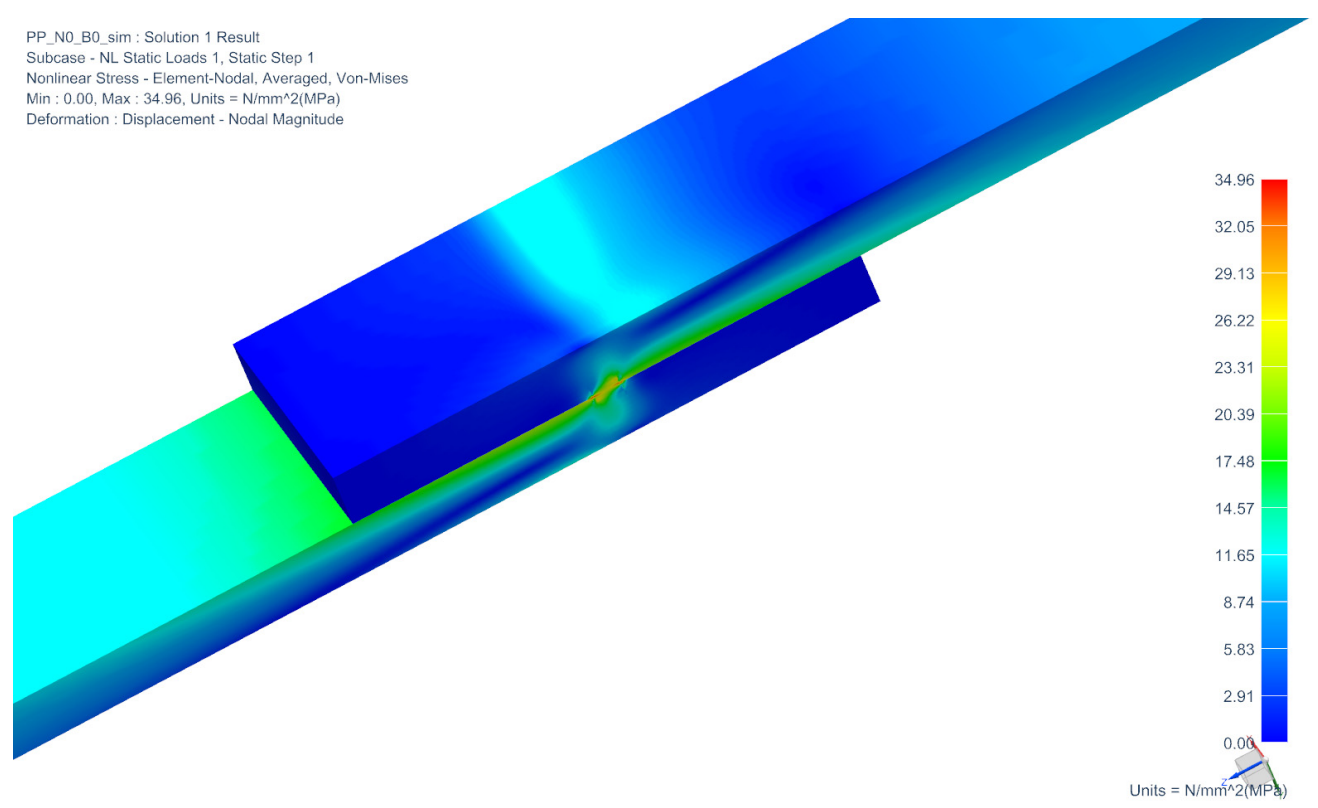

Figure 2. Typical von Mises stress distribution of loaded welded bars for all combinations. Maximal values are in the welded area inside the model and thus can't be seen

Maximal values of stress (equal to material strength) and displacement always occur on the borders of welded area or closely outside. There is a notch, sudden change of cross-section, which always causes concentration of stress. This is the place where the crack probably arises and spreads into the weld. If the welded area is too large then the crack spreads along the borders and the bar is broken outside the weld joint, which sometimes happened during the shear tests. On the fig. 3 there is a von Mises stress in the welded area and surroundings.

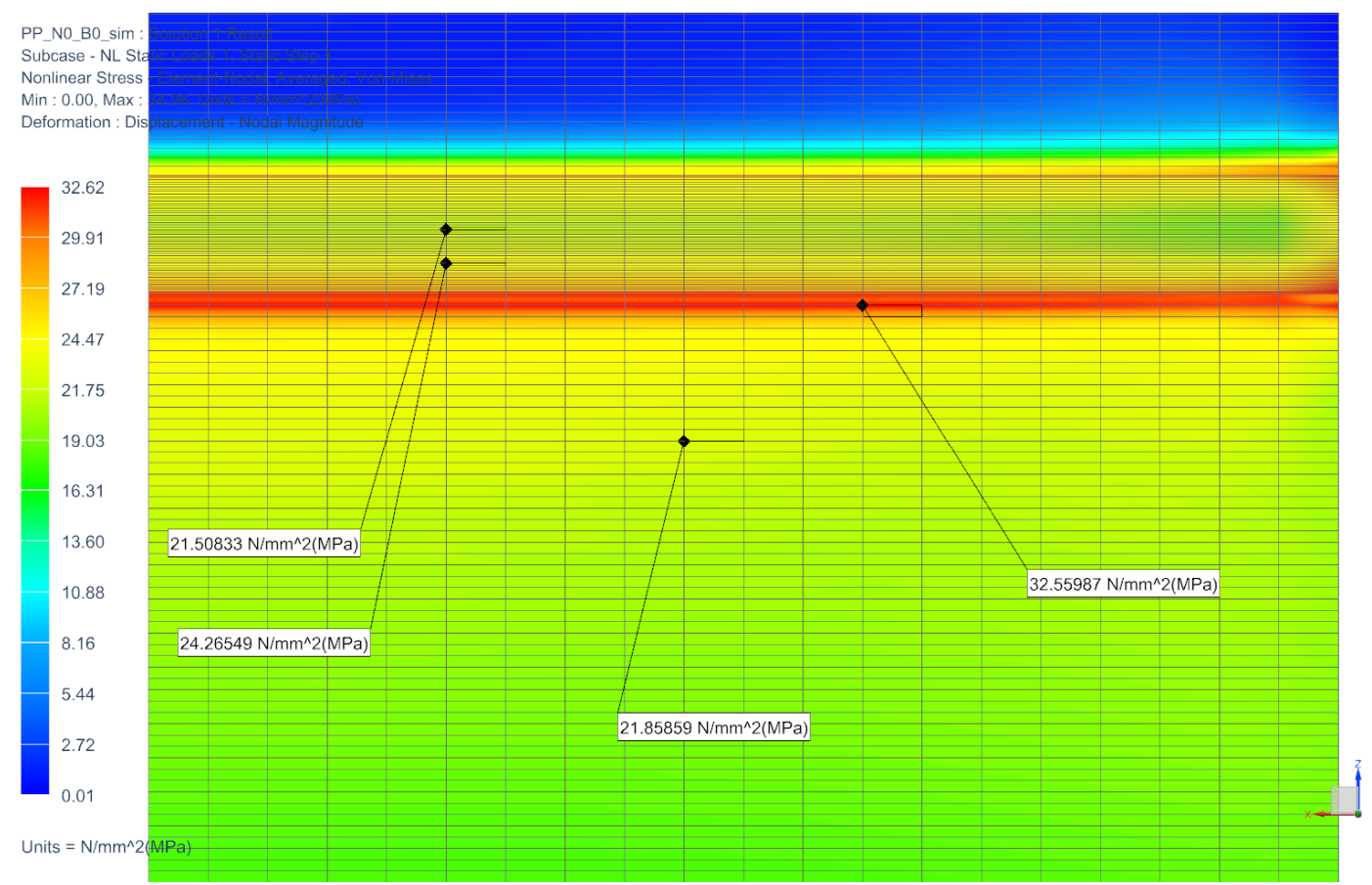

Figure 3. Von Mises stress in the welded area (fine mesh) and surroundings 


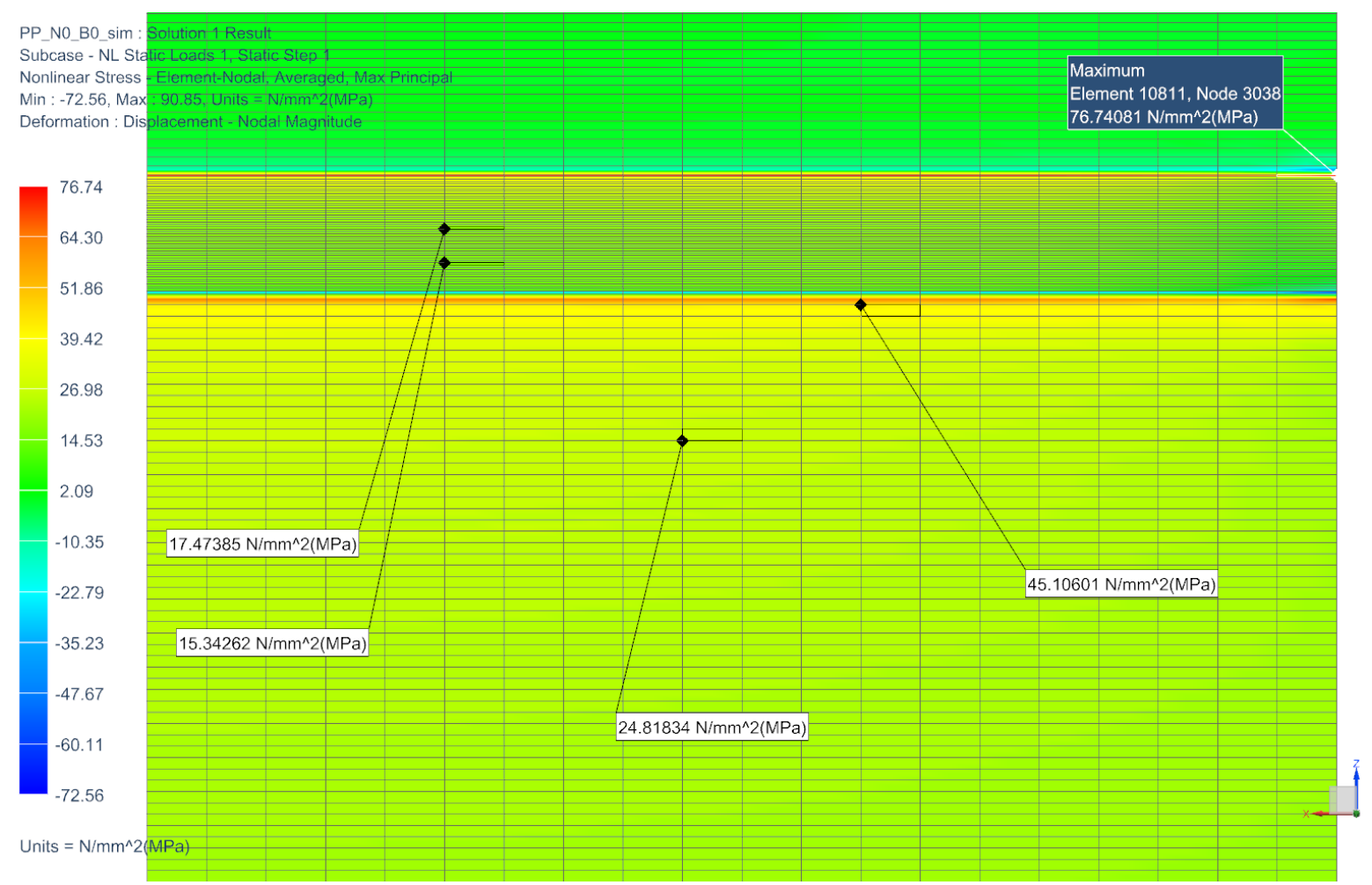

Figure 4. Maximal principal stress in the welded area and surroundings

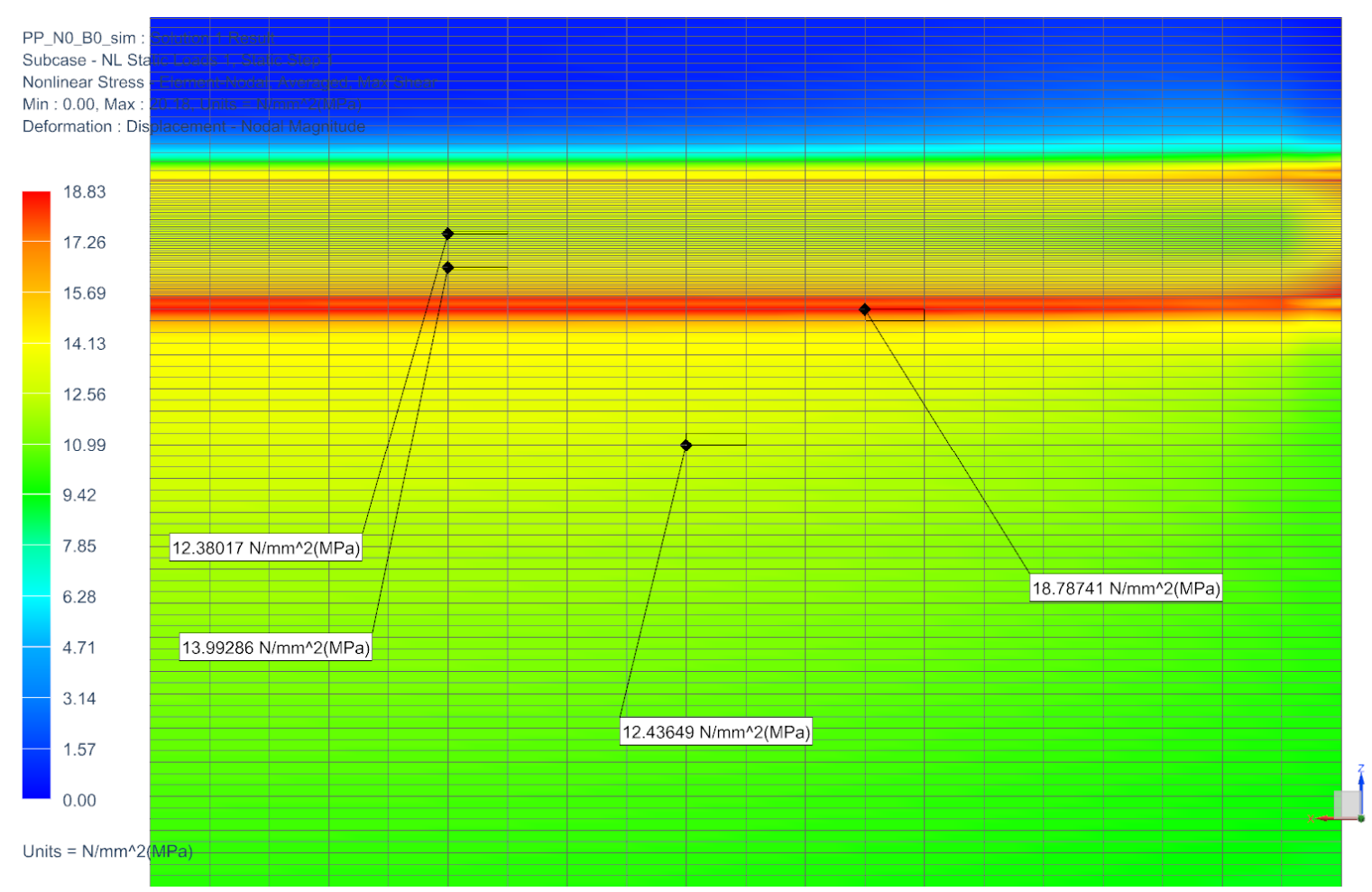

Figure 5: Maximal shear stress in the welded area and surroundings

Mean value of maximal measured stress was 16.9 MPa (see table 3). Von Mises stress inside the welded area was $22 \mathrm{MPa}$ and $33 \mathrm{MPa}$ on the borders. Shear stress values were also important because the performed experiments were shear tests. The shear stress inside the welded area was $12 \mathrm{MPa}$ and $19 \mathrm{MPa}$ on the borders which is in good 
agreement with the measured data (figure 5). Tensile stress is usually responsible for the origin of crack and its spreading so the maximal principal stress is shown on the fig. 4.

\section{Discussion}

For a comparison only two quantities were chosen - von Mises stress inside the welded area and shear stress on the borders (figure 6). Shear stress values were closer to the measured stress for combinations with neat materials (deviation $25 \%$ ). These values had the deviation larger (about $66 \%$ ) for combinations with both reinforced welded plastics. Much more stable were von Mises stress values which were about 15-30\% higher than maximal measured stress for all PP and PC combinations.
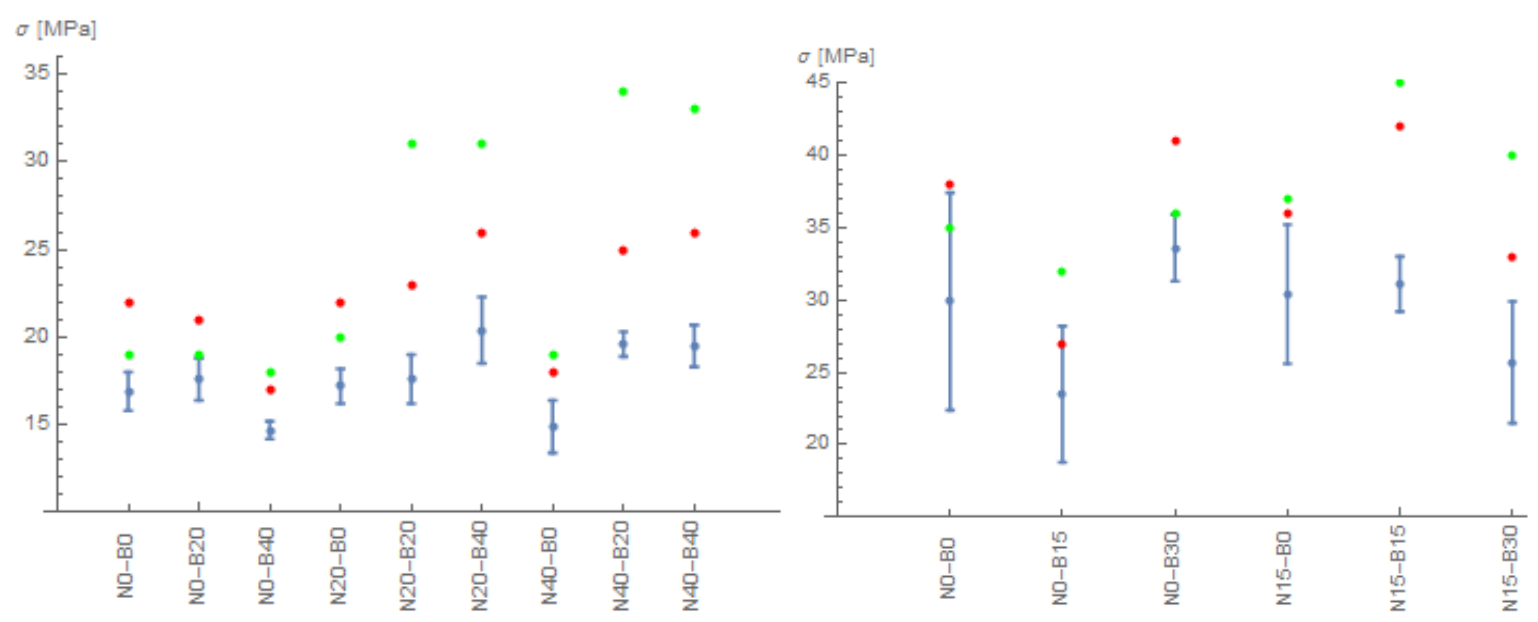

Figure 6. Comparison of simulation results with measured data for welded PP plastics (left) and PC plastics (right). Mean value with $95 \%$ confidence interval of maximal measured stress (blue), maximal shear stress on the welded area borders (green) and von Mises stress inside the welded area (red)

\section{Conclusion}

The design of welded joint of PP and PC composites based on the FEM simulation should be done carefully. Data obtained by tensile test had large variance of measured values so the material model used in simulations is only approximate. The most proper method for determination of weld joint strength is to evaluate von Mises stress inside the welded area (fig. 6). The rupture of the weld joint comes when the evaluated von Mises stress exceeds $35 \%$ of material strength of welded PP composite or $40 \%$ of welded PC composite. During the design process it is necessary to have more strict requirements for safe design.

\section{Acknowledgments}

The publication was created with the support of the project LO1213 - Excellent Engineering Research, the grantor MŠMT, beneficiary - VÚTS, a.s., in support of the National Sustainability Program, with the financial contribution of the Ministry of Education, Youth and Sports of the Czech Republic.

\section{References}

Al-Wohoush, M. H., \& Kamal, M. R. (2012). Characterization of thermoplastic laser-welded joints. International polymer processing, 27(5), 574-583.

Colegrove, P., Ikeagu, C., Thistlethwaite, A., Williams, S., Nagy, T., Suder, W., ... \& Pirling, T. (2009). Welding process impact on residual stress and distortion. Science and Technology of Welding and Joining, 14(8), 717-725.

Costa, A. P. D., Botelho, E. C., Costa, M. L., Narita, N. E., \& Tarpani, J. R. (2012). A review of welding technologies for thermoplastic composites in aerospace applications. Journal of Aerospace Technology and Management, 4(3), 255-265.

Deepak Kumar, M., Karthik, V., \& Venkatakrishnan, P. G. (2016) Ultrasonic plastic welding of glass fiber reinforced plastics. International Journal of Innovative Studies in Sciences and Engineering Technology, 2(5), $25-27$. 
Grewell, D., \& Benatar, A. (2007). Welding of plastics: Fundamentals and new developments. International Polymer Processing, 22(1), 43-60.

He, X. (2012). Finite element analysis of laser welding: A state of art review. Materials and Manufacturing Processes, 27(2).

Mackerle, J. (2001). FEM and BEM analysis and modelling of residual stresses: A bibliography (1998-1999). Finite Elements in Analysis and Design, 37(3), 253-262.

Papazoglou, V. J., Masubuchi, K., Goncalves, E., \& Imakita, A. (1982). Residual stresses due to welding: computer-aided analysis of their formation and consequences. Transactions-Society of Naval Architects and Marine Engineers, 90, 365-390.

Stavrov, D., \& Bersee, H. E. N. (2005). Resistance welding of thermoplastic composites - an overview. Composites Part A: Applied Science and Manufacturing, 36(1), 39-54.

Votrubec, V., Hisem, P., Vinšová, L., \& Lukášová, V. (2017, November). Strength of laser welded joints of polypropylene composites. In IOP Conference Series: Materials Science and Engineering (Vol. 264, No. 1, p. 012015). IOP Publishing.

Yaghi, A. \& Becker, A. (2004). State of the art review - weld simulation using finite elements methods. Technical report, University of Nottingham, UK.

\section{Copyrights}

Copyright for this article is retained by the author(s), with first publication rights granted to the journal.

This is an open-access article distributed under the terms and conditions of the Creative Commons Attribution license (http://creativecommons.org/licenses/by/4.0/). 